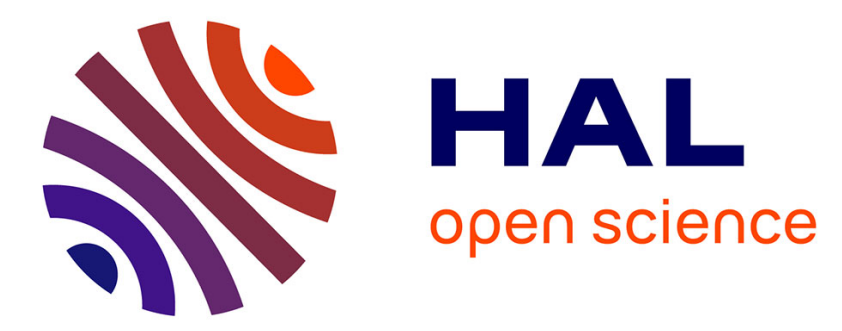

\title{
Optical camera communications link using an LED-coupled illuminating optical fiber
}

Shivani Rajendra Teli, Klara Eollosova, Stanislav Zvanovec, Zabih

Ghassemlooy, Matej Komanec

\section{- To cite this version:}

Shivani Rajendra Teli, Klara Eollosova, Stanislav Zvanovec, Zabih Ghassemlooy, Matej Komanec. Optical camera communications link using an LED-coupled illuminating optical fiber. Optics Letters, 2021, 46 (11), pp.2622. 10.1364/OL.428077 . hal-03341360

\section{HAL Id: hal-03341360 \\ https://hal-amu.archives-ouvertes.fr/hal-03341360}

Submitted on 13 Sep 2021

HAL is a multi-disciplinary open access archive for the deposit and dissemination of scientific research documents, whether they are published or not. The documents may come from teaching and research institutions in France or abroad, or from public or private research centers.
L'archive ouverte pluridisciplinaire HAL, est destinée au dépôt et à la diffusion de documents scientifiques de niveau recherche, publiés ou non, émanant des établissements d'enseignement et de recherche français ou étrangers, des laboratoires publics ou privés. 


\title{
An optical camera communications link using a LED- coupled illuminating optical fiber
}

\author{
Shivani Rajendra Teli, ${ }^{1,}{ }^{*}$ KLara Eollosova, ${ }^{1}$ Stanislav ZVAnOVeC, ${ }^{1}$ Zabih \\ GHASSEMLOOY² AND MATEJ KOMANEC ${ }^{1}$
}

\author{
${ }^{1}$ Faculty of Electrical Engineering, Czech Technical University in Prague, Prague, Czech Republic \\ ${ }^{2}$ Optical Communication Research Group, Faculty of Engineering and Environment, Northumbria University Newcastle, UK \\ *Corresponding author: telishiv@fel.cvut.cz
}

Received XX Month XXXX; revised XX Month, XXXX; accepted XX Month XXXX; posted XX Month XXXX (Doc. ID XXXXX); published XX Month XXXX

\begin{abstract}
In this Letter, we propose and demonstrate a novel wireless communications link using an illuminating optical fiber as a transmitter (Tx) in optical camera communications. We demonstrate an indoor proof-of-concept system using an illuminating plastic optical fiber coupled with a light emitting diode and a commercial camera as the Tx and the receiver, respectively. For the first time, to the best of our knowledge, we experimentally demonstrate flicker-free wireless transmission within the off-axis camera rotation angle range of $0-45^{\circ}$ and the modulation frequencies of 300 and $500 \mathrm{~Hz}$. We also show that, a reception success rate of $100 \%$ is achieved for the camera exposure and gain of $200 \mu \mathrm{s}$ and $25 \mathrm{~dB}$, respectively.
\end{abstract}

Optical camera communications (OCC) and visible light communications (VLC) utilizing light emitting diodes (LEDs) as a transmitter (Tx) and photodetectors (PDs)/cameras as a receiver $(\mathrm{Rx})$, respectively offer functionalities of vision, data communications, and localization, which can be used in many Internet of things (IoT) applications [1, 2]. The major advantages of using complementary metal-oxide semiconductor (CMOS)-based camera Rx are: (i) availability and advancements of smartphones and surveillance cameras in indoor environments such as shopping malls, hospitals, offices, etc.; (ii) higher signal to noise ratio due to longer exposure time $t_{F}$ and larger size photosensitive area that helps to increase the transmission distance $d$; and (iii) the rolling shutter (RS) property of CMOS-based camera, which integrates light in row-by-row manner similar to scanning function. Therefore, the RS-based camera offers data rates $R_{b}$ higher than the frame rate $f_{R}$ of the camera [3].

The fiber-optic lighting is emerging as an alternative to discrete illumination fixtures and semi-discrete LED stripes [4-6]. Typically, plastic optical fibers (POFs) are used for illumination [6] due to their low-cost and easy light coupling using high-power light sources such as Xenon or halogen lamps and LEDs [7, 8]. Recently, silica-based fiber-optic illuminator has emerged, which uses laser light sources due to their small diameters [9]. These illuminating optical fibers, which are easy to bend, can be used for illumination in a range of applications such as IoT, interior designs, shopping centers, aircrafts, fashion, health and safety, etc. These applications thrive on the fiber low-cost (especially in the case of POFs), low weight, easy bending and their mechanical properties. Moreover, it can support low data rates (i.e., few bits/s to kbits/s) indoor OCC-based IoT applications [2]. In this Letter, we introduce a novel concept of OCC that uses an illuminating POF as the Tx; termed as illuminating optical fiber-based OCC (OF-OCC). The intensity modulated (IM) white LED is used to couple the light into the illuminating POF, which basically acts as a long length optical antenna, for illumination, data communications, indoor localization, and sensing.

A camera-based Rx is used to capture the length $d_{f}$ of the illuminating POF for extracting data information. We consider the impact of the off-axis angular orientation $\theta$ of the camera on data capturing and detection over $d_{f}$. This transmission setup resembles the indoor dynamic, mobility, and multicasting scenarios for transmission of low $R_{b}$ information. The lab-scale measurements are carried out in two sections: (i) optical and electrical characterization of the LED measured directly and LED-coupled POF as a radiating Tx; and (ii) data capturing considering $\theta$ of the camera-based $\mathrm{Rx}$. We analyze the quality of the captured data in terms of the success rate of received bits for a range of $\theta$ with respect to $t_{F}$ and the camera gain $G_{v}$. The proposed OF-OCC scheme, to the best of authors knowledge, is the first experimentalbased study on OCC link using LED-coupled illuminating POF.

We have used a $1 \mathrm{~m}$ long polymethyl methacrylate (PMMA) POF with a $3.6 \mathrm{~dB} / \mathrm{m}$ attenuation (measured by the cut-back technique). The core and cladding diameters were 1.6 and $2 \mathrm{~mm}$, respectively (see measured real dimensions in Fig. 1(a)) and the core refractive index of 1.46, and a numerical aperture of $\sim 0.50$. Both ends of POF were cut and polished. Light from a cold white LED (LA CW20WP6, Light Avenue) of a size $500 \times 500 \mu^{2}$ was directly coupled from one end of POF using a 5D stage (3D micromovement stage Thorlabs, MAX313D/M with pitch and yaw tilt platform Thorlabs, APY002/M). The light from the other end of POF was captured using a silica PD and power meter (Thorlabs, 
S121C, wavelength range of 400-1100 nm) for monitoring of LED-coupling performance.

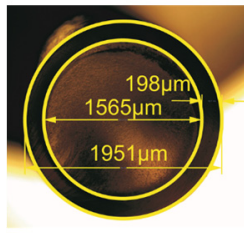

(a)

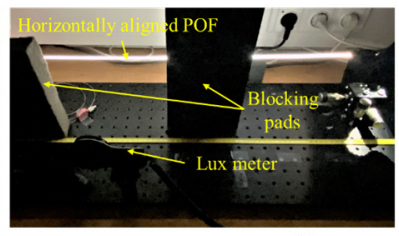

(b)

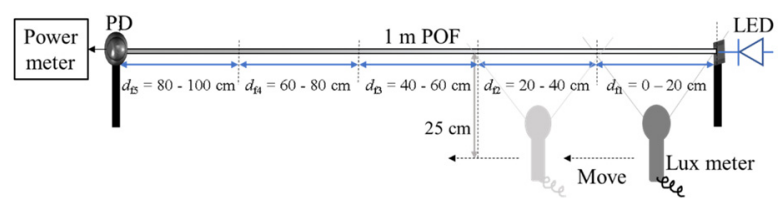

(c)

Fig. 1. (a) Microscope photograph of the used PMMA POF with measured core and cladding dimensions, including the cladding thickness, (b) Experiment setup for POF characterization of $L-I-V$ and illumination pattern, and (c) characterization over $d_{f}$.

At first, we optimize the coupling of light from the LED to the POF by monitoring the optical power level at the end of POF, which was about $11 \mathrm{dBm}$ for the LED at its maximum current. Based on the POF attenuation, which was measured by the cut-back technique to be $3.6 \mathrm{~dB} / \mathrm{m}$, and the LED total output power of $19 \mathrm{dBm}$ the coupling efficiency was estimated to be close to $40 \%$. Figures 1(b) and (c) show the experiment setups for measuring (i) optical power-currentvoltage $(L-I-V)$ characteristics using a lux meter (Voltcraft MS-200LED, $\pm 3 \%$ accuracy); and (ii) the radiation profile of POF. Next, the lux meter at $25 \mathrm{~cm}$ from the POF was used to measure the power profiles along the POF, which is split into segments of $20 \mathrm{~cm}$ long $\left(d_{f 1}-d_{f 5}=(0: 20: 100) \mathrm{cm}\right)$ starting from the LED coupling as illustrated in Fig. 1(c).
Figure 2(a) depicts the measured $L-I-V$ characteristics of the illuminating POF for a range of $d_{f}$. Also shown is the $L-I-V$ plot of the LED measured directly at a distance of $25 \mathrm{~cm}$ that depicts similar optical characteristics to those of commonly used LED sources in VLC systems. Note, $(i)$ the drop in the illumination level with respect to the increasing $d_{f}$; as expected; and (ii) linear $L-I$ plots i.e., a wide dynamic range and thus higher signal to noise ratio, which is highly desirable in IM-VLC systems.

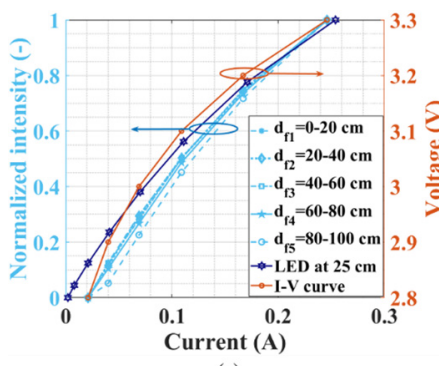

(a)

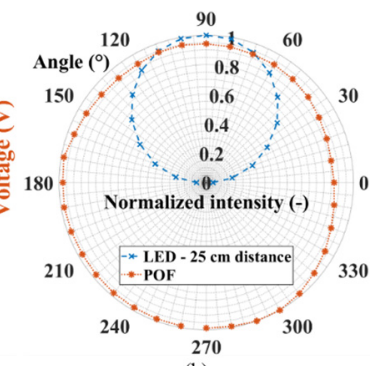

(b)
Fig. 2. POF and LED characterization: (a) L-I-V curves over $d_{f}$ and (b) radiation pattern.

The optical radiation pattern of the POF was measured to obtain the spatial intensity distribution over $360^{\circ}$ to illustrate radiation uniformity as depicted in Fig. 2(b). Note, the illuminating pattern represents a closed hemisphere with a slight deviation of $\sim 10 \%$ from the maximum normalized intensity for angles of +30 to $-30^{\circ}$. This small variation is due to possible fiber bending, random tensions, and contractions over the POF length. Also shown is the radiation profile for the LED measured at a distance of $25 \mathrm{~cm}$, which represents a complete hemisphere (i.e., very close to Lambertian emitter with the order of 1 ).

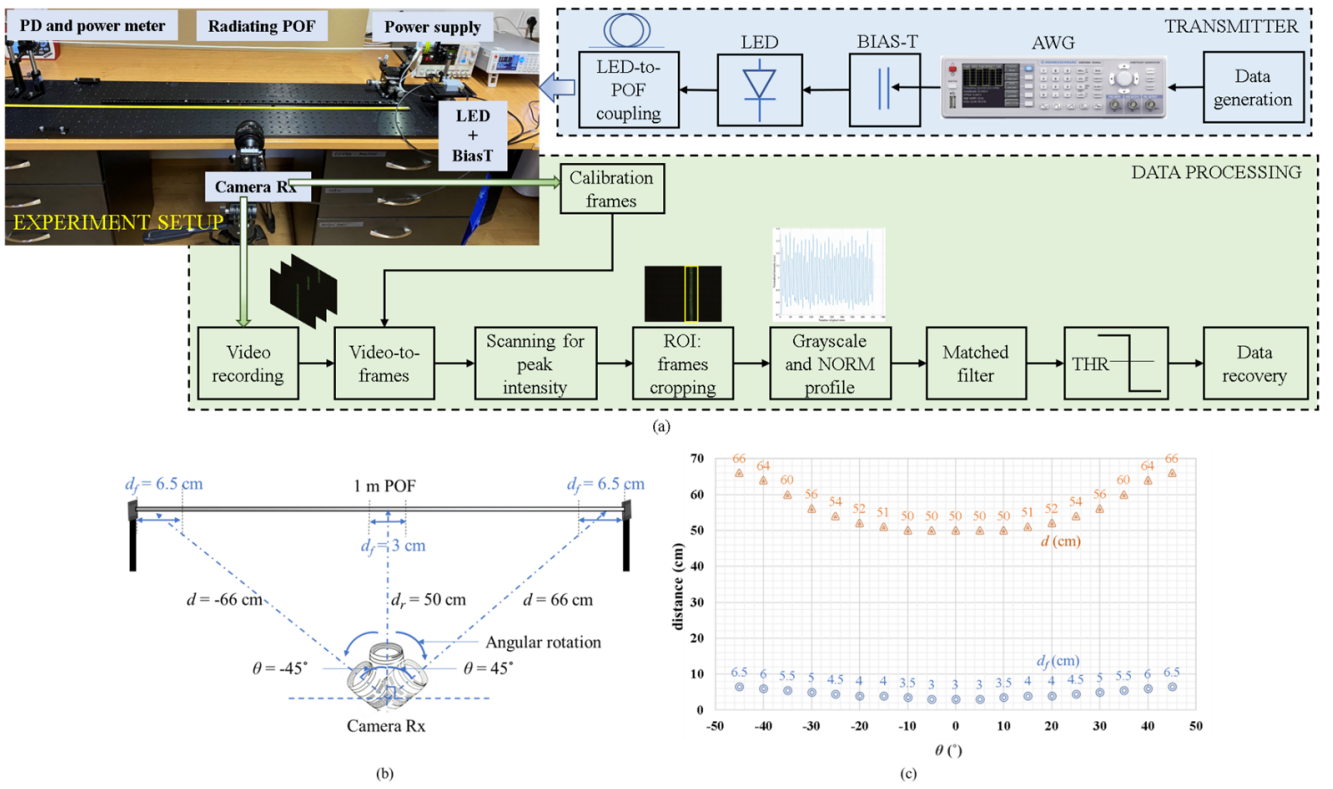

Fig. 3. OCC over POF: (a) data reception flowchart, (b) schematic of data capturing, and (c) variation of $d$ and $d_{f}$ with respect to $\theta$. 

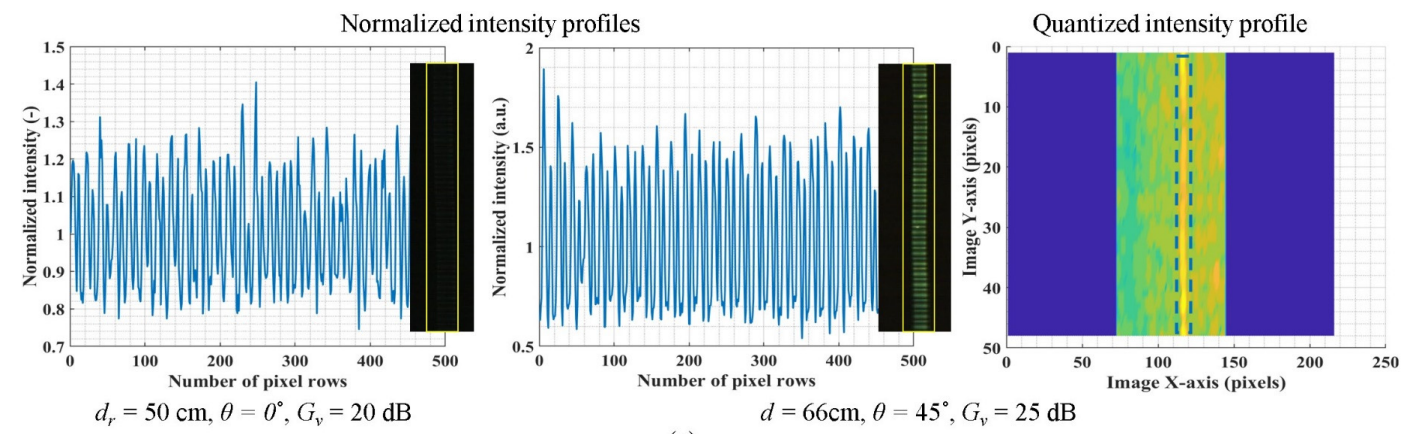

(a)
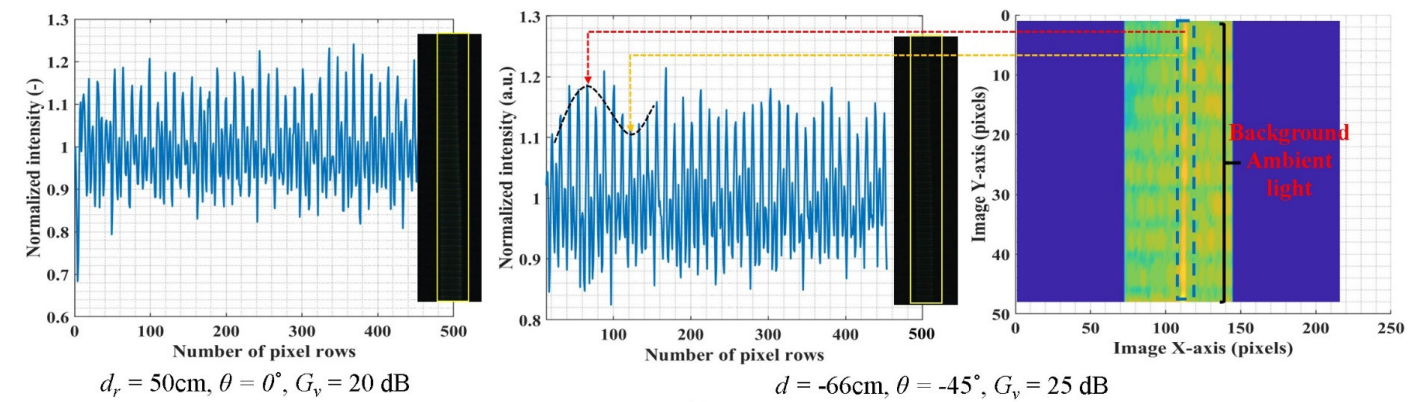

(b)

Fig. 4. Examples of captured illuminating POF at: (a) $f_{s}=300 \mathrm{~Hz}$ and $t_{F}=200 \mu \mathrm{s}$, and (b) $f_{s}=500 \mathrm{~Hz}$ and $t_{F}=400 \mu$ s. Note, yellow and red arrows highlight the intensity variations due to background ambient light.

\begin{tabular}{|c|c|}
\hline Parameter & Value \\
\hline $\begin{array}{l}\text { LED bias current } I_{b} \text { and } \\
\text { supply voltage }\end{array}$ & $300 \mathrm{~mA}$ and $3.3 \mathrm{~V}$ \\
\hline Bias-T & BT-A11 \\
\hline $\mathbf{R x}$ & IC capture USB 2.0 camera \\
\hline Resolution & $648 \times 484$ pixels \\
\hline$f_{R}$ & $25 \mathrm{fps}$ \\
\hline$t_{F}$ & $200,400 \mu \mathrm{s}$ \\
\hline$G_{v}$ & $25,20,15 \mathrm{~dB}$ \\
\hline$d_{r}$ (radial distance) & $50 \mathrm{~cm}$ (from center of POF) \\
\hline & $66 \mathrm{~cm}$ (center-to-ends of POF) \\
\hline$f_{s}($ modulation frequency $)$ & $300,500 \mathrm{~Hz}$ \\
\hline $\boldsymbol{\theta}$ & $-45^{\circ}>0^{\circ}>45^{\circ}$ \\
\hline Data packet size & $6 \mathrm{~b} /$ packet $[110010]$ \\
\hline
\end{tabular}

The aim of the proposed scheme is to investigate OF-OCC system using illuminating POF as the Tx and a camera as the $\mathrm{Rx}$, see Fig. 3(a). A non-return-to-zero (NRZ) on-off-keying $(\mathrm{OOK})$ data format (most commonly used in OCC) generated in MATLAB and uploaded to an arbitrary waveform generator (AWG Rohde \& Schwarz HMF2550 $50 \mathrm{MHz}$ ) is used for IM of the LED (biased at $I_{b}$ of $300 \mathrm{~mA}$ ) via the bias-T (BTA11). The IM light is coupled into the POF. During all experiments, LED was covered by a black shield in order to capture only signal from the illuminating POF. Table 1 shows all the key experiment parameters adopted in this work.

A CMOS RS-based camera positioned at the center of the POF at the radial distance $d_{r}$ of $50 \mathrm{~cm}$ and with a total rotation angle $\theta$ of $\pm 45^{\circ}$, see Fig. 3(b), captures the intensity modulated illumination. Note that, the actual transmission distance $d$ and $d_{f}$ vary with $\theta$; see Fig. 3(c). Note, all the measurements are performed in indoor ambient light conditions. The camera set to capture in RS mode sequentially integrates all illuminated pixels at the exposure time, which is the same as scanning $[10,11]$. In RS-based cameras, the readout time ensures that there is no overlapping of the rows of pixels, thus allowing multiple exposures of a single captured image. This feature offers simultaneous capturing of multiple incoming light states in a single frame as each row is exposed once to the light as well as flicker-free operation $[10,11]$. Considering that the illuminating intensity of POF is low and reduces further with $d_{f}$, see Fig. 2(a); for the camera we used $t_{F}$ of 200 and $400 \mu \mathrm{s}$ and $G_{v}$ of 15,20 , and $25 \mathrm{~dB}$. The OCC data processing was performed using traditional image processing techniques. Both the recorded data and calibration video streams containing the captured signal and the POF template shape and intensity compensation, respectively are divided into image frames prior to frame-by-frame processing to decode the received data. Following frame division, peak intensity scanning is performed to locate the captured POF in the image frame and calculate the region-of-interest (ROI) to obtain the coordinates, see Fig. 3(a), which define the ROI boundaries of a full captured frame [12]. Next, the ROI cropped images are converted from RGB to Grayscale to retrieve the intensity profile. Finally, the transmitted data stream is regenerated using matched filtering, thresholding, and binarization of the data frames by converting them into a vector transformation.

The current proof-of-concept experiments were performed under the ambient light, where we measured the background light intensity of $150 \mathrm{~lx}( \pm 3 \mathrm{~lx})$ from the ceiling lamps. Measurements were carried out for $d, t_{F}, \theta$, and $G_{v}$, see 
Table 1. The data packet of 6-bit [110010] was generated and transmitted at $f_{s}$ of 300 and $500 \mathrm{~Hz}$ using a repeat packet strategy to improve the link performance. Figure 4 shows the examples of captured image frames and their normalized and quantized intensity profiles. The intensity profiles play an important role in determining the higher and lower intensities representing 1 and 0 bits in the received image frames for further thresholding and demodulation. It can be seen that, the intensity profile improves with (i) increasing $G_{v}$, which enhances signal amplification while passing through the camera ADC prior to being focused on the IS [3]. This is due to the fact that, $G_{v}$ presents the software-defined global gain of both the IS and the column amplifier block, which is given as $G_{v}(\mathrm{~dB})=20 \log _{10}\left[V_{\mathrm{ADC}} / V_{\text {pixels }}\right]$, where $V_{\mathrm{ADC}}$ is the voltage value, which is sampled by the ADC, and $V_{\text {pixles }}$ is the voltage obtained from the pixel integration of light during the exposure time. Therefore, higher $G_{v}$ mitigates the influence of ambient light on the integrity of data reception; and (ii) reducing $t_{F}$. Also captured are the intensity profiles having an interferometric shape due to the ambient light, which is captured together with the light from the illuminating POF.

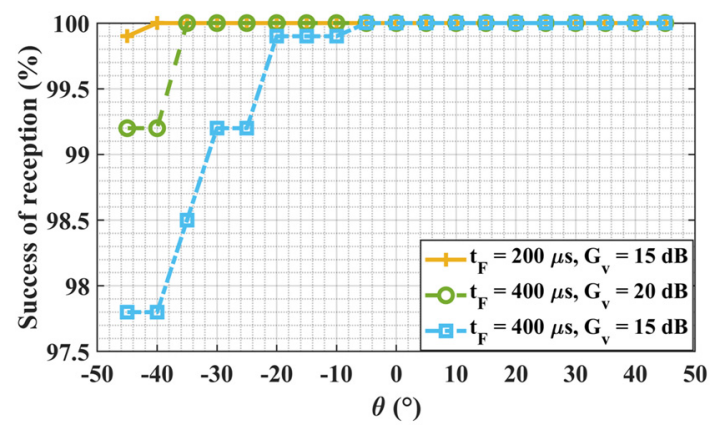

(a)

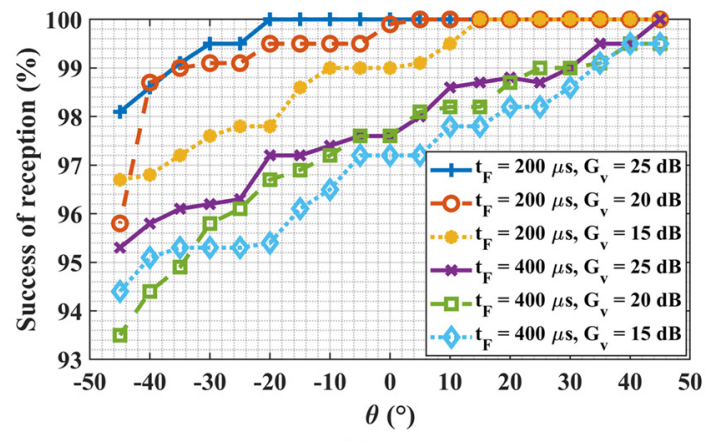

(b)

Fig. 5. Performance of OF-OCC: the success of reception with respect to $\theta$ for $f_{s}$ of: (a) $300 \mathrm{~Hz}$, and (b) $500 \mathrm{~Hz}$

At $\theta$ of $0^{\circ}$ and $45^{\circ}, 21-36$ and 23-39 repeated data packets were captured in every image frame at $f_{s}$ of 300 and $500 \mathrm{~Hz}$, respectively. Considering that, only a small number of bits is transmitted, the OCC link performance is analyzed in terms of the reception success, which is defined as the ratio of incorrectly decoded bits to the total number of transmitted bits. Figure 5 shows the performance of the proposed OF-OCC link in terms of the reception success rate. It can be seen from Fig. 5(a) that, for $f_{s}$ of $300 \mathrm{~Hz}$ (i) $100 \%$ (i.e., error-free transmission) reception success rates are achieved at $\theta$ of $40^{\circ},-35^{\circ}$, and $-5^{\circ}$ for $t_{F}$ of 200 and $400 \mu$ s and $G_{v}$ of 15 and $25 \mathrm{~dB}$, respectively; and (ii) the reception success rates decrease below the $\theta$ values mentioned in (i) (i.e., near the end of POF) with the lowest value of $97 \%$ at $t_{F}$ of $400 \mu \mathrm{s}$ and $G_{v}$ of $15 \mathrm{~dB}$. For $f_{s}$ of $500 \mathrm{~Hz}$, the reception success rates of $>93 \%$ are achieved for $\theta>-45^{\circ}$ for all values of $t_{F}$ and $G_{v}$, which has increased to $>97 \%$ when closer to the LED, see Fig. 5(b). Note, the performance of the OCC link improves when the camera is closer to the LED irrespective of $\theta$ and $d$. This is due to the increasing illumination levels as seen from the $L-I-V$ characteristics in Fig. 2(a) and the intensity profiles in Fig. 4.

In this Letter, we proposed and experimentally demonstrated a novel concept of OCC system using an illuminating POF as the Tx and a camera as the Rx. We evaluated the proposed OF-OCC system for range of camera orientation angles and showed that, despite the small diameter of POF, flicker-free transmission with $100 \%$ reception success rates were possible. It is envisioned that, the proposed study can be further expanded by increasing $d$ and $f_{s}$ and by optimizing $G_{v}$ and $t_{F}$ to overcome noise effect in different environments and thereby improve the reception success rates, which was not the focus of this letter. This first proof-of-concept result paves the way for new OF-OCC systems capable of transmitting low data rates information as part of IoT in smart environments.

Funding. H2020 Marie Sklodowska-Curie Innovative Training Network (VisIoN 764461), EU COST Action CA19111 (NEWFOCUS), and Technologická Agentura České Republiky (TA ČR) (FW01010571).

Disclosures. The authors declare no conflicts of interest.

\section{References}

1. H. Aksu, L. Babun, M. Conti, G. Tolomei and A. S. Uluagac, IEEE Communications Magazine, 56, 138 (2018).

2. S. R. Teli, S. Zvanovec and Z. Ghassemlooy, IEEE International Conference on Internet of Things and Intelligence System (IOTAIS), Bali, 40 (2018).

3. T. Le, N. Le and Y. M. Jang, 2015 International Conference on Information and Communication Technology Convergence (ICTC), Jeju, 124, (2015).

4. V. Guerra, C. Guerra, J. Rabadan, R. Perez-Jimenez and B. Ortega, 12th International Symposium on Communication Systems, Networks and Digital Signal Processing (CSNDSP). IEEE, (2020).

5. D. Křemenáková, J. Militký, B. Meryová, and V. Lédl, World Journal of Engineering (2013).

6. J. Huang, D. Křemenáková, J. Militký, and G. Zhu, Autex Research Journal, 15, 13, (2015).

7. J. Yang, Z. Liu, B. Xue, Z. Liao, L. Feng, N. Zhang, J. Wang, and J. Li, IEEE Photonics Journal, 10, 1, (2018).

8. Y. Wang, S. Su, G. Zhang, G. Sun, J. Zhang, J. Wang, and Y. Ren, Optical Precision Manufacturing, Testing, and Applications. 10847. International Society for Optics and Photonics, (2018).

9. W. S. Klubben, S. L. Logunov, E. J. Fewkes, J. Mooney, P. M. Then, P. G. Wigley, H. Schreiber, K. Matias, C. J. Wilson and M. Ocampo, Proc. SPIE 9702, Optical Fibers and Sensors for Medical Diagnostics and Treatment Applications XVI, 970218, (2016).

10. T. Kuroda, Essential principles of image sensors, 2017.

11. S. R. Teli, S. Zvanovec, R. Perez-Jimenez, and Z. Ghassemlooy, Appl. Opt. 59, 10357, (2020).

12. A. Duque, R. Stanica, H. Rivano, A. Desportes, Computer Communications, 160, 52, (2020). 


\section{Full references}

1. H. Aksu, L. Babun, M. Conti, G. Tolomei and A. S. Uluagac, "Advertising in the loT Era: Vision and Challenges," IEEE Communications Magazine, vol. 56, no. 11, pp. 138-144, 2018.

2. S. R. Teli, S. Zvanovec and Z. Ghassemlooy, "Optical Internet of Things within 5G: Applications and Challenges," 2018 IEEE International Conference on Internet of Things and Intelligence System (IOTAIS), Bali, pp. 40-45, 2018.

3. T. Le, N. Le and Y. M. Jang, "Performance of rolling shutter and global shutter camera in optical camera communications," 2015 International Conference on Information and Communication Technology Convergence (ICTC), Jeju, 2015, pp. 124-128.

4. V. Guerra, C. Guerra, J. Rabadan, R. Perez-Jimenez and B. Ortega, "Analysis of Illumination-Fiber-to-Wireless Links," 2020 12th International Symposium on Communication Systems, Networks and Digital Signal Processing (CSNDSP). IEEE, 2020.

5. D. Křemenáková, J. Militký, B. Meryová, and V. Lédl, "Characterization of side emitting plastic optical fibers light intensity loss," World Journal of Engineering (2013).

6. J. Huang, D. Křemenáková, J. Militký, and G. Zhu, "Evaluation of illumination intensity of plastic optical fibres with TiO2 particles by laser treatment," Autex Research Journal vol. 15, no. 1, pp. 13-18, 2015.

7. J. Yang, Z. Liu, B. Xue, Z. Liao, L. Feng, N. Zhang, J. Wang, and J. Li, "Highly uniform white light-based visible light communication using red, green, and blue laser diodes," IEEE Photonics Journal, vol. 10, no. 2, pp. 1-8, April 2018.

8. Y. Wang, S. Su, G. Zhang, G. Sun, J. Zhang, J. Wang, and Y. Ren, “Fiber coupling technology of white LED," in Optical Precision Manufacturing, Testing, and Applications, J. McBride, J. Tan, S. Han, and X. Zhang, Eds., vol. 10847, International Society for Optics and Photonics. SPIE, 2018, pp. $139-146$.

9. W. S. Klubben, S. L. Logunov, E. J. Fewkes, J. Mooney, P. M. Then, P. G. Wigley, H. Schreiber, K. Matias, C. J. Wilson and M. Ocampo "Novel light diffusing fiber for use in medical applications," Proc. SPIE 9702, Optical Fibers and Sensors for Medical Diagnostics and Treatment Applications XVI, 970218, 2016.

10. T. Kuroda, "Essential principles of image sensors," CRC press, 2017.

11. S. R. Teli, S. Zvanovec, R. Perez-Jimenez, and Z. Ghassemlooy, "Spatial frequency-based angular behavior of a short-range flicker-free MIMOOCC link," Appl. Opt. 59, 10357-10368, 2020.

12. A. Duque, R. Stanica, H. Rivano, A. Desportes, "Analytical and simulation tools for optical camera communications," Computer Communications, vol. 160, pp. 52-62, 2020. 